Int. J. Electrochem. Sci., 15 (2020) $8363-8374$

\title{
Electrosynthesis and Spectroelectrochemistry Properties of Copolymers Based on 3,4-Ethylenedioxythiophene and Quaterthiophenen Derivatives
}

\author{
Lanlan Shen ${ }^{1,2}$, Zhiru Bai ${ }^{2}$, Yu Jiang ${ }^{2}$, Liying Li $^{1}$, Fengxing Jiang ${ }^{1, *}$, Jingkun Xu ${ }^{1}$, Huanhuan Qiu ${ }^{1}$, \\ Huixuan Liu ${ }^{1}$, Rongri Tan ${ }^{1, *}$ \\ ${ }^{1}$ Department of Physics, Jiangxi Science and Technology Normal University, Nanchang 330013, P. R. \\ China. \\ 2 Jiangxi Engineering Laboratory of Waterborne Coatings, Jiangxi Science and Technology Normal \\ University, Nanchang 330013, P. R. China. \\ *E-mail: f.x.jiang@live.cn (F. Jiang) and rogertanr@hotmail.com (R. Tan)
}

doi: $10.20964 / 2020.09 .73$

Received: 3 January 2020 / Accepted: 15 July 2020 / Published: 10 August 2020

Based on quaterthiophenen, bisethyleneoxyl are appended to thiophene rings (2,5-bis(2,3dihydrothieno[3,4-b][1,4]dioxin-5-yl)thieno[3,2-b]thiophene, BED-TT) for modified monomer structure and obtain a lower onset potential as well as strong electron donor properties. Different molar ratio copolymers of BED-TT with 3,4-ethylenedioxythiophene (EDOT) was electrochemically synthesized, and the effects of changes in the amount of EDOT on the electrochemical and spectrochemistry properties of the copolymers were tracked. The resultant copolymer P(BED-TT-coEDOT) was characterized via cyclic voltammetry, Raman, and spectroelecrochemistry. The study found that the amount of EDOT in the mixed system of BED-TT and EDOT can regulate the oxidation potential, electrochemical activity, and redox process of polymer films to a certain extent. The spectroelectrochemical results revealed that the copolymer P(BED-TT-co-EDOT) has a narrower optical band gap (1.63-1.67eV) and a richer electrochromic color (from pink purple, purple to gray blue, blue).

Keywords: quaterthiophenen; EDOT; copolymer; electrochemistry; spectroelecrochemistry;

\section{$\underline{\text { FULL TEXT }}$}

(C) 2020 The Authors. Published by ESG (www.electrochemsci.org). This article is an open access article distributed under the terms and conditions of the Creative Commons Attribution license (http://creativecommons.org/licenses/by/4.0/). 